\title{
A Thermodynamic Analysis of Energy Flow in Optical Fiber Communication Systems
}

\author{
Salam Abdelhady ${ }^{1}$ \\ ${ }^{1}$ Department Canadian International College, CIC, Cairo, Egypt \\ Correspondence: Salam Abdelhady, Professor of Energy Systems, Engineering Department Canadian \\ International College, CIC, Cairo, Egypt. E-mail: salama_hady@cic-cairo.com
}

Received: May 21, 2012 Accepted: June 5, 2012 Online Published: July 15, 2012

doi:10.5539/apr.v4n3p22 URL: http://dx.doi.org/10.5539/apr.v4n3p22

\begin{abstract}
A thermodynamic analysis of energy flow in different elements of an optical fiber communication system is presented. The analysis depends on a previously introduced entropy approach that postulated the flow of electric charges as a flow of electromagnetic waves. Such a postulate is introduced here to find a plausible explanation of the electro-optic Kerr effect. Following the same approach, the transmitter and receivers of the optical communication-systems are explained according to measurement results as converters of electric waves into light waves or converters of light waves into electric waves. Similarly, pumping of optical energy or electrical energy into the elements of a communication system is found equivalent to pumping of entropy. Such results are introduced to derive energy equations that model the flow of energy and different processes in an optical communication-system. The introduced approach that depends on the postulated definition of electric current is compared to a classical approach in an analysis of energy flow into $80 \mathrm{MHz}$ fiber laser.
\end{abstract}

Keywords: Kerr effect, photoelectric effect, Light Emitting Diodes, entropy, attenuation, dispersion and absorption

\section{Introduction}

Most literature of physics considers the electric current as a flow of electrons. Such a concept has led to the confusing duality-property of electrons as the electric current is a form of energy or waves and it is not a flow of electrons (Abdelhady, 2011a). In this study, a previously introduced fundamental equation that embraces the flow of electric charges and magnetic flux is applied to perform a thermodynamic analysis of different components and processes in the optical communication systems (Abdelhady, 2011a). This equation defines the nature of electric current and magnetic flux as a flow of electromagnetic waves that have a specific potential or as a flow of entropy by virtue of their potentials (Abdelhady, 2010a). Such an approach found, in a previous study, the photoelectric effect as conversion of light waves into electric current (Abdelhady, 2011b).

Kerr effect is investigated in this study to prove the applicability of the introduced entropy approach in optical systems (Haaiday, Resnick, \& Walker, 2004). Accordingly, such an approach is tried to find a plausible explanation for available measurement-results of characteristics of the light emitting diodes as a transmitter and the photocell as a receiver in the optical fiber communication systems. To express the advantage of the introduced approach, this approach and a traditionally used classical-approach are applied on a case study. Finally, concluding results will be introduced

\section{A Study of Energy Flow in Optical Fiber Systems}

The electromagnetic waves are characterized by Maxwell's equations (Haaiday, Resnick, \& Walker, 2004). However, the time in Maxwell's equation was replaced by entropy, as it is a property of time alone (Zeh, 1990). So, the electric and magnetic components of the electromagnetic waves were represented into space-entropy coordinates instead of traditionally used space-time coordinates (Abdelhady, 2010a). According to similarity of the equations characterizing the flow of heat and electric charges and to the results of some experiments, the flow of electric charges was postulated as a flow of electromagnetic waves whose electric component is oscillating around a specified electric potential into the space-entropy coordinates (Abdelhady, 2010a).

Heat flow is defined thermodynamically as the temperature times the rate of entropy flow according to following equation (Yunus \& Michael, 2006): 


$$
\dot{Q}_{\text {thermal }}=T \dot{S}_{t h}
$$

By analogy, the electric current was derived in a previous study as the product of the electric potential "E" times the rate of entropy flow " $\dot{S}$ " (Abdelhady, 2011b):

$$
\dot{Q}_{\text {elect }}=E \dot{S}_{\text {el }}
$$

In a previous analysis, the unit of $\dot{S}$ in equations (1) and (2) was defined in Watt/Volt (Abdelhady, 2010b, 2010c).

The previous concepts were introduced to derive a fundamental equation of thermodynamics that embraces the flow of heat and electric charge (Abdelhady, 2010a). Applying such an equation on the elements of optical fiber-communication systems which are subjected to flow of electric and thermal (or optical) energy only, the equation will be reduced to:

$$
\dot{U}=\dot{Q}_{\text {thermal }}+\dot{Q}_{\text {elect }}
$$

Equation (3) expresses the rate of change of the stored energy in an element of an optical-fiber system as the sum of the flows of heat and electric charges. Substituting the values $\dot{Q}_{\text {thermal }}$ and $\dot{Q}_{\text {elect }}$ from equations (1) and (2) into equation (3), the final form of the energy equation will be:

$$
\dot{U}=T \dot{S}_{t h}+E \dot{S}_{e l}
$$

\section{Kerr Effect}

The Kerr effect, also called the quadratic electro-optic effect (QEO effect), is defined in literature as a change in the refractive index of a material in response to an applied electric field (Haaiday, Resnick, \& Walker, 2004) The postulated nature of the electric charge as electromagnetic waves represent an approach to a plausible explanation of such increase when it is subjected to an electric field according to the following equation that characterizes the nonlinear behavior of the refractive index of cores of optical fibers (Lin, Painter, \& Agrawal, 2007):

$$
n=n_{0}+n_{2} * P / A_{e f f}
$$

Where: $\mathrm{n}_{0}$ is the refractive index of the fiber core at low optical power levels, $\mathrm{n}_{2}$ is the nonlinear refractive index coefficient $\left(2.35 \times 10^{-20} \mathrm{~m}^{2} / \mathrm{W}\right.$ for silica), $\mathrm{P}$ is the power of the flowing light or electromagnetic waves in Watts and $A_{e f f}$ is the effective area of the fiber core in square meters. According to equation (5), when the core of an optical fiber is subjected to higher power of the flowing electromagnetic waves, such power "P" will increase the refractive index "n." When applying electric field on the optical fibers, it will impose a flow of electric current or ionized electromagnetic waves through such fibers (Abdelhady, 2011b). Accordingly, Kerr effect is plausibly explained as result of applying electric field when considering pumping of electric power is pumping of electromagnetic waves that will increase the power of the flowing electromagnetic waves through the optical fibers. Such explanation sustains also the approach we are going to follow in the following sections when analyzing the energy flow through optical-fiber communication systems.

\section{Plausible Explanation of the Processes in LEDs and Photocells}

The process of optical detection depends on the photoelectric effect where the incident light generates a flow of electric charges (Haaiday, Resnick, \& Walker, 2004). According to a previous study, the photoelectric effect was defined as a process of ionizing the incident light during its reflection on a metal plate in the photocells (Abdelhady, 2011b). The reflected light gains after reflection a specific potential that depends on its frequency and on the electrode potential of the reflecting metal plate (Abdelhady, 2011b). Figure 1 shows the measured stopping potential which is equal to the potential of the reflected photocurrent as a function of the incident light-frequency for Sodium as the reflecting metal-plate $[3,10]$. The slope of this plot equal to Planck's constant " $\mathrm{h}=6.626 * 10^{-34} \mathrm{~J} . \mathrm{s}$ ". Such results can be represented by the following relation between the frequency of the incident light " $v_{\text {incident }}$ " and the potential "V" of the reflected waves or photons after ionization by the metal plate (Abdelhady, 2011b):

$$
h * v_{\text {incident }}=e * V
$$

The symbol "e" in equation (6) indicates the charge of the reflected photons $\left(\mathrm{e}=1.6 * 10^{-19} \mathrm{Joule} / \mathrm{Volt}\right)$. As seen in Figure (1); the frequency of the light that may be converted into an electric current and gains after reflection the minimum potential of " $V_{\text {minimum }} \cong 1.8$ Volts" should be higher than the "threshold frequency $v_{O}$ " (Keiser, 
2000). Such minimum threshold frequency depends on the electrode potential of the metal of reflector plate in the considered cell.

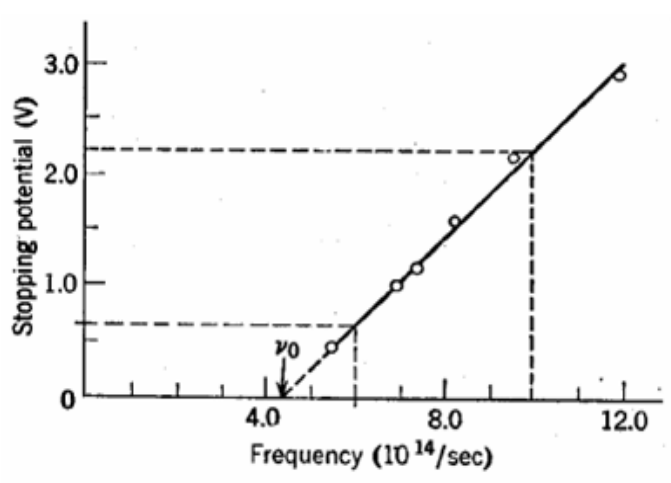

Figure 1. Plot of the measured stopping voltage as a function of frequency for sodium plate (taken from Millikan's Nobel Prize winning experiment)

Similar measurement results are found also in the light emitting diodes that assure the conversion of an electric current, as ionized electromagnetic waves, into light waves. Figure 2 shows the measured frequency of emitted light from a diode as a function of the applied potential for different types of diodes. Increasing the potential drop across such LED's led to increasing the frequency of the emitted light too according to the equation (Hackworth, 2000; Ducharme,1999):

$$
e * V=h *\left(\frac{c}{\lambda_{\text {emitted }}}\right)=h * v_{\text {emitted }}
$$

In equation (7), "c $=300 * 10^{6} \mathrm{~m} / \mathrm{s}$ " is the speed of light and " $\lambda$ " is the wavelength of the emitted light in meters. The measured slope of the curve in Figure 2 determines the same value of Planck's constant as found from the measured slope in Figure 1 for photocells. So, the postulated definition of the flow of electric charges as a flow of electromagnetic waves finds also a plausible explanation for such measurement results. The process in the LED may be explained according to such postulate as de-ionizing the incident electric current such that it is converted into light of a frequency that satisfies equation (7). In other words, the built-in potential of each LED's p-n junction de-ionizes, according to its energy gap, the incident electric current and converts it into light waves of zero potential.

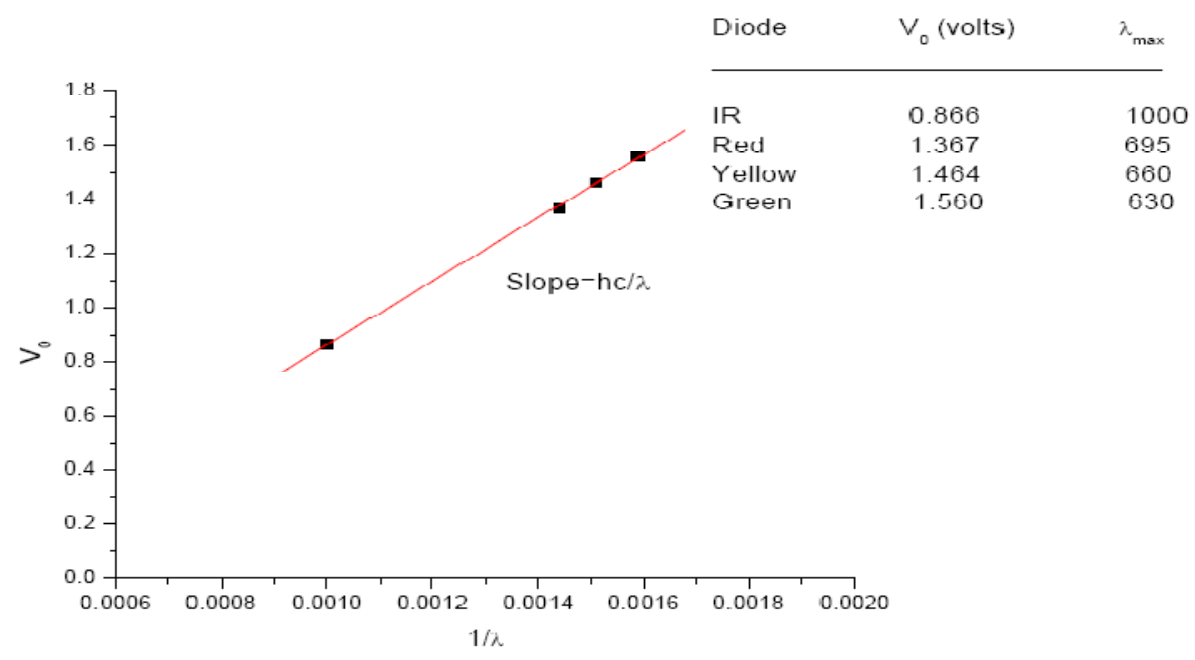

Figure 2. Determination of Planck's constant using LED's (Csele, 2004) 
According to the measurement results in Figure (2), for each diode there is a minimum potential of the flowing current, called threshold potential " $V_{\text {thresh }}$ or $\mathrm{V}_{0}$ " that led to emit light from such diode. Figure 1 and Figure 2 clearly show the correspondence between the processes in the LEDs and photocells. The same slopes of both lines and the correspondence between the threshold frequency in Figure 1 and the threshold potential in Figure 2 indicate that the processes in the diodes and the photocells are process of ionizing and de-ionizing the light waves or photons. So, the availability of emitters and transmitters that have the same frequency bands is not a matter of chance as both are working with the same waves but proceeding in opposite directions. Equations (6) and (7) can be simplified by one proportionality equation as follows:

$$
\lambda_{\text {incident or emitted }} \propto \frac{1}{V_{\text {photocell or diode }}}
$$

Equation (8) is similar to the following Wien's law of thermal radiation (Yunus \& Michael, 2006).

$$
\lambda_{\max } \propto \frac{1}{T_{\text {black body }}}
$$

\section{Energy Analysis of Diodes and Lasers}

Figure (3) shows a diode circuit where the input electric-energy is emitted from the diode as optical or infra-red waves (Csele, 2004). Applying the energy equation (4) on such diode:

$$
\dot{U}_{\text {diode }}=\left(E \dot{S}_{\text {el }}\right)_{\text {input }}-\left(T \dot{S}_{\text {th }}\right)_{\text {output }}
$$

In equation (10), the first term in the R.H.S. represents the energy input or the input power to the diode and the last term in the R.H.S. represents the energy output from the diode in the form of optical or infrared waves. The difference between these two terms, $\dot{U}_{\text {diode }}$, represents the rate of increase of diode's internal energy that is lost to surroundings.

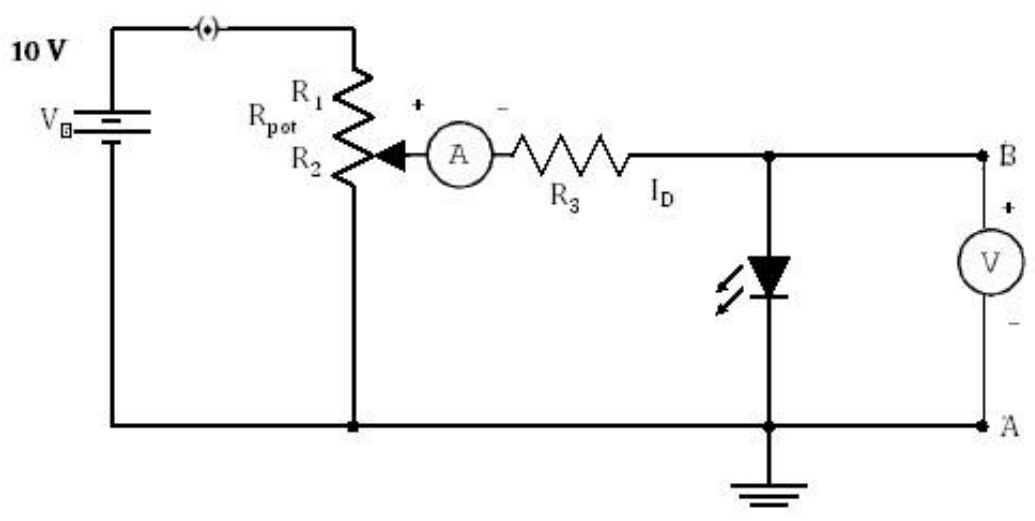

Figure 3. A typical Diode Circuit (ASTM, 1993)

The rated power of the diode is denoted $\dot{W}_{\text {diode }}$ and it represents its energy input and can be substituted in the following equation to find the rate of flow of entropy into the diode's circuit :

$$
\dot{W}_{\text {inputdiode }}=E \dot{S}_{\text {el }}
$$

For a typical diode, the values of the input power and rated potential are as follows (Csele, 2004):

$$
\dot{W}_{\text {diode }}=36 \mathrm{~mW}, E=1.8 \text { Volt }
$$

Substituting these values in equation (12), we get:

$$
\dot{S}_{e l}=0.02 \mathrm{Watt} / \text { Volt }
$$

For an ideal diode, it is possible to neglect the energy losses in the diode;

$$
\dot{U}_{\text {diode }}=\left(E \dot{S}_{\text {el }}\right)_{\text {input }}-\left(T \dot{S}_{\text {th }}\right)_{\text {output }}=0
$$

Hence, 


$$
\left(E \dot{S}_{\text {el }}\right)_{\text {input }}=\left(T \dot{S}_{\text {th }}\right)_{\text {output }}
$$

The Right Hand Side of equation (12) defines the input radiation to the optical fibers as the product of the entropy flow through the fiber times the temperature of the optical fiber. The potential of the electric energy is measured directly by a standard voltmeter; however, the scale of measurement of temperature in volts by thermocouples depends on the kind of the introduced thermocouple. So, it is possible to adopt a reference scale such that the increase in the flow electric entropy, by electric energy input, will be equivalent to the increase of the flow of thermal entropy. According to equation (12), such condition implies the following equality:

$$
E_{\text {diode }} \equiv \widehat{T}_{\text {fiber }}
$$

For a type $\mathrm{T}$ thermocouple, the normal absolute temperature of fibers with respect to a reference junction at absolute zero can be found from the corresponding tables as follows (ASTM, 1993),

$$
\bar{T}_{T . C .}=12 \mathrm{~m} . \mathrm{V}
$$

To satisfy equation (13); the following scaling up of the temperature scale in optical fibers should be introduced:

$$
\widehat{T}_{\text {fiber }}=150 \bar{T}_{\text {T.C. }}
$$

\section{Energy Flow in Optical Fiber Systems}

The used light in optical fibers is a part of the "electromagnetic spectrum" that includes also x-rays, ultraviolet radiation, microwaves, radio, TV, cell phones, and all the other wireless signals. However, the used wavelengths in optical fibers are determined by two factors: longer wavelengths in the infrared for lower loss in the glass fiber and at wavelengths which are between the absorption bands of the selected diodes or lasers (Hüfner, 2003). Thus the optimally required wavelengths are 850,1300 and $1550 \mathrm{~nm}$. Such wavelengths belong to typical thermal radiation and are available, as previously discussed, in the traditionally used transmitters, as LEDs, and receivers, as photo-detectors. Hence, it is possible to apply the laws of thermodynamics, equation (7), on an optical fiber that receives the output of a diode " $\left(T \dot{S}_{\text {th }}\right)_{\text {output }}$ " as follows::

$$
\dot{U}_{\text {fiber }}=T d \dot{S}
$$

Differentiating both sides of equation (15) with respect to the length of the fiber "z", we get:

$$
\frac{d \dot{U}_{\text {fiber }}}{d z}=T \frac{d \dot{S}}{d z}
$$

The decreasing flow of energy into the fibers by absorption can be expressed by the following equation (Kapron et al., 1970):

$$
\frac{d \dot{U}_{\text {fiber }}}{d z}=-\alpha T d \dot{S}
$$

Where $\alpha$ is the absorption coefficient of the fiber material per unit length .

From equations (16) and (17), we get:

$$
T \frac{d \dot{S}}{d z}=-\alpha T d \dot{S}
$$

Solving the differential equation (18), we get:

$$
\dot{S}(z)=\dot{S}_{z=0} e^{-\propto z}
$$

Introducing these results into the energy equation (10), it is possible to get the following equation (Ira Jacobs, 1995):

$$
\int_{0}^{L}-\alpha T \dot{S}(z) d Z=\sum \dot{W}_{\text {input }}+\sum \dot{W}_{\text {pumped }}-\sum \dot{W}_{\text {output }}
$$

The value of input, output or pumped power " $\dot{W}$ " can be estimated according to the nature of power, optical or electrical, according to the following equations:

$$
\begin{aligned}
\dot{W}_{\text {thermal }} & =\hat{T} \dot{S}_{\text {thermal }} \\
\dot{W}_{\text {electrical }} & =E \dot{S}_{\text {electrical }}
\end{aligned}
$$


Equation (18) can be also applied to the optical fibers for each frequency-band as an approach to analyze the amplification process at each band of wavelengths (Ira Jacobs, 1995).

\section{A Case Study}

The previous analysis of energy flow into optical fibers, which is defined by equation (20), is based on the postulated definition of electric current as a flow of electromagnetic waves that have an electrical potential (Abdelhady, 2011a). Equation (20) expresses the input, output and dissipated energies in a fiber-communication system as superposition of two modes of electromagnetic waves; the propagating light and the pumped electromagnetic waves that have an electrical potential.

However, the classical approach to the analysis of energy flow in optical fibers considers the electric current as a flow of electrons of a duality nature or a wave-particle nature. This approach proposes an envelope of such confusing wave-particles that are called solitary waves or solitons and imposes the use of the Schroedinger equation as an obligatory mathematical model to involve such duality. In most literature, such solitons propagate within a waveguide which size varies with the assumed potential of such flow (Bale et al., 2009). The soliton is the solution of the Schroedinger equation with the addition of a nonlinear term, mostly the Kerr nonlinearity, which originates from the interaction of the light waves with the assumed propagating electrons. Such model of solution is based also on de Broglie's original wave-particle concept, which is based on the Special theory of relativity (Bale et al., 2009).

To compare the two approaches, a case study of $80 \mathrm{MHz}$ fiber laser, shown in Figure 4, is considered (Nielsen, 2006).

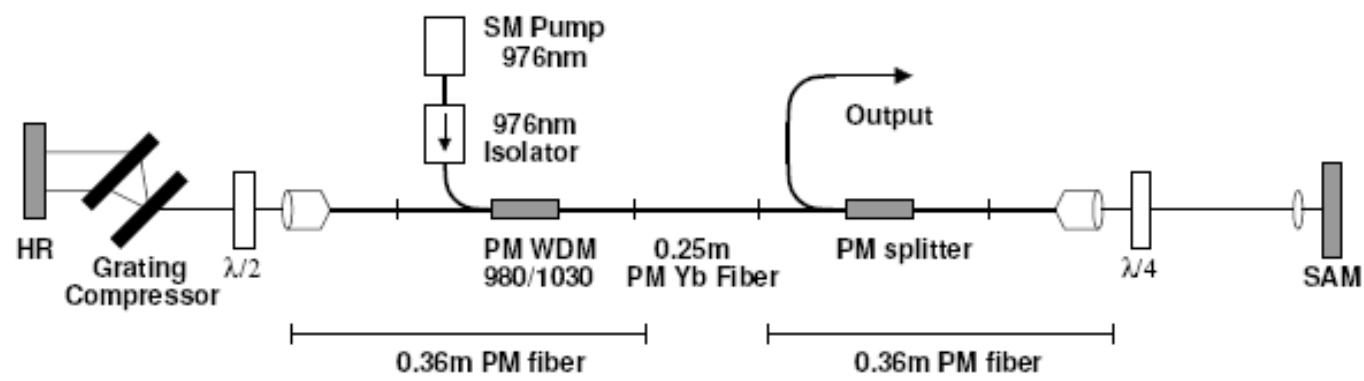

Figure 4. Diagram of the fiber-laser configuration

Details of the shown system are explained in the Ph.D. thesis of C.S. Nielsen (Nielsen, 2006). As the knowledge about the dispersion in a fiber is often of key importance, analysis of such dispersion by the two approaches is considered too. In his thesis, Nielsen (Nielsen, 2006) introduced a numerical model based on the nonlinear Schroedinger equation (NLSE) for simulation of the nonlinear losses and cavity losses in general. He found through his model the temporal shape of a pulse for different phase shifts (Nielsen, 2006). Other investigators found by using similar models the relation between the different values of transmitted and the incident intensities, dependence of dispersion parameters on the frequency of the flowing light and the phase shifts and dependence of the intensities on defined distances (Xu, 2003; Rebane, 2004).

However, more general results can be achieved through the entropy approach by direct superposition of the flowing light and electric current considering both as electromagnetic waves according to the following equation (Orfanidis, 2004).

$$
I(\omega)=\left|E_{1}\right|^{2}+\left|E_{2}\right|^{2}+2 E_{1} E_{2} \cos \left(\phi_{2}(\omega)-\phi_{1}(\omega)\right)
$$

$\mathrm{E}_{1}$ and $\mathrm{E}_{2}$ are the time independent complex electric field amplitudes of the electric current and flowing light. In this equation; the flow of electric charges is considered as a wave of amplitude $\mathrm{E}_{1}$ that oscillate around a non-zero potential, the potential of the pumped energy, and $\mathrm{E}_{2}$ as the amplitude of the propagating light where both may have the same frequency $(\omega)$ but of different phase shifts: $\phi_{1}(\omega)$ and $\phi_{2}(\omega)$. Attenuation along the optical fiber that influences on the pulse propagation in the fiber can be implemented directly by numerical integration of equation (20) and introducing equation (19) along the fiber length including the transmitters, pumping laser-diodes, detectors and receivers. Such results cannot be easily attained by the use of the NLSE as it is limited by the definition of the electric current as a flow of electrons and cannot deal with the diodes and 
detectors as reversible converters of light and electric power. Terms of equation (20) may be involved to find the relevance of the output power to input power by substituting the main parameters of the optical fiber, lasers and detectors of the experimental setup used in the thesis (Nielsen, 2006).

\section{Conclusion}

Following previously introduced postulates that define the flow of charge as a flow of entropy, the energy flow through elements of an optical fiber communicattion system was analysed. Kerr effect was intially analyzed to prove the applicability of such approach in optical systems. It is found that the process of converting of light into electric current is a reversible process which can be processed in both directions, but, by different mechanisms. Such approach simplifies the calculation of energy pumping and different processes in different elements of optical fibers as of their thermal or electrical potentials. The postulated approach is applied on a laser fiber to estimate dispersion and energy flow in such fiber to show the advantages of such approach with respect to the classical approach that considers the flow of electric current as a flow of electrons.

\section{References}

Abdelhady, S. (2010a). A Fundamental Equation of Thermodynamics that Embraces Electrical and Magnetic Potentials. J. Electromagnetic Analysis \& Applications, 2, 162-166.

Abdelhady, S. (2010b). Comments Concerning Measurements and Equations in Electromagnetism. $J$. Electromagnetic Analysis \& Applications, 2, 677-678.

Abdelhady, S. (2010c). An Approach to a Universal System of Units. J. Electromagnetic Analysis \& Applications, 2, 549-556.

Abdelhady, S. (2011a). An Entropy-Approach to the Duality Property. J. Electromagnetic Analysis \& Applications, 3, pp. 220-227.

Abdelhady, S. (2011b). Comments on Einstein's Explanation of Electrons, Photons, and the Photo-Electric Effect. Applied Physics Research, 3(2), pp. 230-240. http://dx.doi.org/10.5539/apr.v3n2p230

American Society for Testing and Materials. (ASTM). (1993). A Manual on the Use of Thermocouples in Temperature Measurement (4th edition). Philadelphia, New York.

Bale, B. G., Boscolo, S., Schwartz, O. Y., \& Turitsyn, S. K. (2009). Localized Waves in Optical Systems with Periodic Dispersion and Nonlinearity Management, Review Article. Advances in Nonlinear Optics, 1-14. http://dx.doi.org/10.1155/2009/181467

Csele, M. (2004). Fundamentals of light sources and lasers. John Wiley \& Sons. http://dx.doi.org/10.1002/0471675210

Ducharme, S. (1999). Measuring Planck's Constant with LEDs. Retrieved form http://physics.unl.edu/directory/ducharme/ducharme.html

Haaiday, D., Resnick, R., \& Walker, J. (2004). Fundamentals of Physics (7th edition). New York: John Wiley \& Sons, 2004.

Hackworth, M. (2000). Measuring Planck's Constant. Retrieved from www.physics.isu.edu/ hackmart/planck's

Hüfner, S. (2003). Photoelectron Spectroscopy Principles and Applications (3rd Ed.). Berlin: Springer.

Ira Jacobs. (1995). Optical Fiber Communication Technology and System Overview. Trends in Optical Fiber Metrology and Standards, NATO ASI Series, Kluer Academic Publishers, 285, 576-591.

Kapron, E. P. et al. (1970). Radiation Losses Glass Optical Waveguides. Appl. Phys. Letter, 17(423), November $15,1970$.

Keiser, G. (2000). Optical Fiber Communication (3rd Ed.). McGraw-Hill, 2000

Lin, Q., Painter, O. J., \& Agrawal, G. P. (2007). Nonlinear optical phenomena in silicon waveguides. Opt. Express, 15, 16604. http://dx.doi.org/10.1364/OE.15.016604

Nielsen, C. K. (2006). Mode Locked Fiber Lasers: Theoretical and Experimental Developments; Ph.D. Thesis. Retrieved from http://users-phys.au.dk/ckn/Files/thesis.pdf, December 2006

Orfanidis, S. J. (2004). Electromagnetic Waves and Antennas. Retrieved from www.ece.rutgers.edu/ orfanidi/ewa

Rebane, L. (2004). Propagation characteristics of coherent optical waves in a stratified medium with Kerr nonlinearity. Ph.D. Thesis. Retrieved from http://cens.ioc.ee/ liis/thesis_liis.pdf 
$\mathrm{Xu}$, B. (2003). Study of Fiber Nonlinear Effects on Fiber Optic Communication Systems. Ph.D. Thesis. Retrieved from http://www.ece.virginia.edu/ optcom/publications/bo/xu_dissertation.pdf

Yunus, C., \& Michael, A. B. (2006). Thermodynamics: An Engineering Approach. McGraw-Hill Science Engineering, 2006.

Zeh, H. D. (1990). The Physical Basis of the Direction of Time. Science, 249(4965), 192-193. 